\title{
Implicit motor learning through observational training in adults and children
}

\author{
ANNIE VINTER and PIERRE PERRUCHET \\ University of Bourgogne, Dijon, France
}

\begin{abstract}
Although evidence of implicit motor learning on the basis of observation alone has been reported, there is some data to suggest that the phenomenon could be contaminated by the intentional exploitation of explicit knowledge. In the present experiment, a special procedure was adapted to study observational learning in a situation involving the acquisition of a new drawing behavior. The participants consisted of adults and children 6-10 years of age. The results provide support for the view that overt motor practice is not strictlynecessary for implicit motor learning. They demonstrate that children display capacities similar to those of adults in this form of learning. Some suggestions are made to account for the contradictory results present in this area of research.
\end{abstract}

Adults can learn implicitly a sequence of aiming movements, as has been shown in serial reaction time studies (e.g., Nissen \& Bullemer, 1987; Stadler, 1989) and continuous target displacement, as has been illustrated in visuomotor pursuit experiments (Pew, 1974; Wulf \& Schmidt, 1997). In both cases, independently of any intention of the participants, motor performance improved selectively, as a function of practice, in response to the structural features of the situation. A priori, improved behavior might be due to the acquisition of knowledge concerning the perceived events, to the acquisition of a chain of motor actions, or to both. A way of addressing this issue consists in examining whether performance still improves when participants have only received observational training. If the suppression of motor responding has no detrimental effect, this would show that the motor component is not necessary. This question has been tackled in the implicit learning literature (e.g., Cohen, Ivry, \& Keele, 1990; Howard, Mutter, \& Howard, 1992; Nattkemper \& Prinz, 1997; Rüsseler \& Rösler, 2000; Stadler, 1989; Willingham, 1999; Willingham, Nissen, \& Bullemer, 1989; Ziessler, 1994), and contradictory results have been reported. One way to reconcile these results might be to consider the possibility that positive evidence of observational learning actually consists of cases in which learning is not really implicit. Although there is little consensus within the literature on what implicit learning is (e.g., Cleeremans, 1993; Perruchet \& Vinter, 1998; Reber, 1993), most definitions specify that the observed behavioral sensitivity to the structural features of the training situation must not be due to an intentional exploitation of subjects' explicit knowledge

Correspondence should be addressed to A. Vinter, L.E.A.D., CNRS 5022, Faculty of Sciences, University of Bourgogne, 6 bv Gabriel, 21000 Dijon, France (e-mail: annie.vinter@u-bourgogne.fr).

-Accepted by previous editorial team about these features. In the case of observational motor learning, some data have suggested that the phenomenon could fail to respect this criterion.

Howard et al. (1992), for instance, found higher levels of awareness in participants learning by observation than in those learning by acting. Likewise, Willingham (1999) reported positive observational learning only in participants who also achieved a high level of explicit knowledge on the relevant learning features. Rüsseler and Rösler (2000) showed that different types of neural activity occurred in explicit or implicit learners in the course of a serial reaction time task in which deviant perceptual or motor fragments were sometimes introduced. Only explicit learners exhibited neural activity in relation to the perceptual deviants. These results provide support for the view that observational learning is mediated by explicit processes. It thus appears crucial, for tackling the question of the possibility of learning implicitly a motor behavior by observation only, to devise a learning procedure in which the "contamination" by explicit influences is strongly avoided. We recently developed such a procedure, called the neutral parameter procedure, which has been implemented in a drawing task. The feature manipulated in the learning task deals with a covariation between where to start and how to progress in a drawing. In spontaneous drawing, people tend to respect the start-rotation principle (Van Sommers, 1984), which states that the selection of a starting point located at the top of a figure (more precisely, above a virtual 11 o'clock-5 o'clock axis in the case of a circle) is predominantly associated with a counterclockwise rotation, whereas a clockwise rotation is mainly associated with a bottom start. The experimental manipulation consists in inverting this covariation. We have shown that appropriately designed motor practice might indeed lead adults and children (Vinter \& Perruchet, 1999, 2000) to modify their spontaneous drawing behavior in such a way that it actually reflects the relevant structural feature manipulated during the learning episode. The originality of the procedure is that there is no 
confusion between the parameter on which unconscious effects are assessed (inversion of the covariation) and the parameter on which task achievement is assessed (accuracy and speed in tracing), which makes a priori any potential explicit knowledge about the manipulation useless.

The aim of the present experiment was to test whether implicit observationallearning can occur when explicit influences on behavior are avoided. We simply adapted our drawing procedure by requiring participants to observe figures being drawn on a monitor screen in a way that either did or did not respect the start-rotation principle. The question was: Could the behavioral change observed after motor practice in earlier experiments be induced by observation alone? We expected that it could, at least in adults. However, the outcome might be different for children. Indeed, some authors claim that, in the course of development, children move from a largely procedural or implicit basis of knowledge to a more declarative or explicit knowledge (Karmiloff-Smith, 1992). This would lead us to expect children's performance to be worse than that of adults. The motor component is thought to have a positive and constructive role in the implicit learning of a sensorimotor task such as drawing because children are considered to be better at gaining procedural knowledge than explicit knowledge. Age differences should thus be observed in observational motor implicit learning. In support of this hypothesis, it is worth pointing out that the single study reporting a deficit in children compared with adults in an implicit learning task used a task based on perceptual judgments without motor involvement (Maybery, Taylor, \& O’Brien-Malone, 1995).

\section{METHOD}

\section{Participants}

A total of 68 right-handed adults (54 females, 14 males) volunteered for the observational training experiment. They were aged 18-29 years (mean age, 21.58 years) and were randomly assigned to one of two groups: a $20 \%$ principle-conformity group $(N=35)$ and an $80 \%$ principle-conformity group $(N=33)$. They were students at the University and were unaware of the purpose of the study. Furthermore, 124 right-handed children ( 71 females, 53 males) also participated in the experiment. They were divided into three age groups (mean age, 6 years, $n=42,25$ females, 17 males, age range, 5 years 10 months to 6 years 4 months; mean age, 8 years, $n=41$, 19 females, 22 males, age range, 7 years 9 months to 8 years 3 months; mean age, 10 years, $n=41,26$ females, 15 males, age range, 9 years 8 months to 10 years 4 months). In each age group, the children were randomly assigned to one of the two training groups: the $80 \%$ or the $20 \%$ principle-con formity group (6 years: $n=21$ in the $80 \%$ group; 8 years: $n=19$ in the $80 \%$ group; 10 years: $n=21$ in the $80 \%$ group). Each age group corresponded to one school level, the youngest children coming from the first grade, and none of the children were educationally advanced or retarded or had visual or psychomotor deficits.

\section{Material}

The material was the same as in the motor-training experiment (Vinter \& Perruchet, 1999). This experiment was run with a Wacom PL-100V tablet (display size: $19.19 \times 14.39 \mathrm{~cm}$ ), which functions both as a digitizer (temporal sampling at $200 \mathrm{~Hz}$ ) and as a monitor screen. It thus provides a natural paper-like interface, with the par- ticipants in the test phase drawing directly on the tablet with a cordless pen over figures whose appearance on the screen was software controlled. The figures were as follows: circle $(1.2 \mathrm{~cm}$ diameter $)$, rectangle $(1.2 \times 2.4 \mathrm{~cm})$, square $(1.2 \times 1.2 \mathrm{~cm})$, and triangle $(1.2 \times$ $1.2 \times 1.2 \mathrm{~cm})$. They were centered inside a square of $4 \times 4 \mathrm{~cm}$ that was permanently displayed in the central region of the tablet. In the learning phase, the figures were drawn on line on the screen at an average speed of $1.4 \mathrm{~cm} / \mathrm{sec}$, so that they usually appeared drawn at a constant velocity. This required a deceleration phase before the tracing of the angle, followed by an acceleration phase after the angle. However, this phase of deceleration-acceleration was sometimes replaced by a constant velocity in the course of the drawing of a random selection of figures ( $25 \%$ of the entire set), which thus seemed to be traced at a changing velocity (Viviani \& Stucchi, 1992). In the test phase, the starting point, which appeared on the figure, had a diameter of $0.1 \mathrm{~cm}$ and was located at the same absolute position, top or bottom, whatever the figure (middle of the horizontals for the square and rectangle, 12 o'clock/6 o'clock positions for the circle, and top of the triangle or middle of its base).

\section{Procedure}

The entire experimental session comprised a training phase, a test phase, and a questionnaire phase. In the training phase, the participants were asked to watch closely as a figure was drawn on the screen in order to judge whether it was drawn at a constant velocity (yes response) or not (no response). This velocity judgment task made the observation of the drawings necessary for the participants. Thus we could be sure that the participants attentively observed the drawing process, including, incidentally, where it started and how it progressed. Figures appeared one at a time at an interval of $400 \mathrm{msec}$ after the experimenter pressed a key following the participant's yes or no response. No time constraint was imposed on the participants for providing the judgment, and no feedback was given concerning their responses. The participants did indeed perceive that most of the figures appeared to be drawn with a constant velocity, although others (around $25 \%$ of the figures) did not. There were no particularly interesting results in this task for our present purposes, and we shall not consider this further here. The participants in each age group were randomly assigned to one of two groups that differed as a function of the percentage of figures traced in conformity with the start-rotation principle (SRP). The first group was presented with a set of figures, $20 \%$ of which respected the SRP (20\% SRP group), and the second group saw a set of figures, $80 \%$ of which respected the principle (80\% SRP group).

The participants saw 40 figures consisting of 10 repetitions of 4 figures (circle, triangle, rectangle, and square). The set of figures presented to the $20 \%$ SRP group included $10 \%$ of the figures (i.e., 4 figures, one exemplar of each basic figure), starting at the top and rotating counterclockwise, and $10 \%$ of figures displaying the reverse version of the SRP, a bottom start combined with a clockwise rotation. The remaining $80 \%$ of the figures violated the SRP, with half of them (16 figures, four repetitions of each basic figure) starting at the top and rotating clockwise, and half starting at the bottom and rotating counterclockwise. The same structure applied to the $80 \%$ SRP group, but the proportions of figures that did or did not conform to the SRP were inverted. Note that there were thus as many top as bottom starts and as many clockwise as counterclockwise rotations for each basic figure. In each group, the figures appeared in a fixed order for a given participant (e.g., 10 series of a succession consisting of a circle, a square, a triangle, and a rectangle), and different orders were randomly used across participants. Moreover, the order in which the various combinations of starting locations and rotation directions appeared was random across the 10 repetitions of each figure.

Whatever the group, a test phase followed after a 5-6 min interval during which the experimenter explained to the participants that they now had to trace over the same types of figures, which would 
appear already drawn on the screen and that they had to respect the indicated starting point, located either at the top or at the bottom of the figure. Nothing was said about the direction of rotation of the drawing movement. Only if explicitly requested did the experimenter specify that the participants were free to adopt any direction of rotation they chose. The instructions required the participants to trace over the figure as accurately and as fast as possible after the emission of a signal beep that announced its appearance on the screen (time interval of $100 \mathrm{msec}$ ). The experimenter told the participants that their drawing velocity would be automatically recorded by the digitizer and analyzed later, as if the aim of the experiment was related to the velocity of drawing. The test comprised 24 figures (six repetitions of each basic figure), half of them presenting a top-starting point and the other half a bottom-starting point. The software recorded the direction of rotation and the starting location used by the participants for each drawing.

In the questionnaire phase, the participants were invited to report anything that they might have noticed during training. They were then given a forced-choice test: When they were asked to remember whether a figure started at the top or the bottom, they had to decide whether the drawing progressed mainly clockwise or counterclockwise. These two judgments were combined in order to provide a unique measure of the participant's awareness of the manipulated covariation between rotation and starting location. Then, the participants were informed of the purpose of the study.

\section{RESULTS}

\section{Implicit Performance}

We wondered whether the fact of having seen figures traced in a certain mode would lead the participants to conform to this mode when they subsequently had to draw the same types of figures. If this were the case, the mean percentages of respect for the principle should differ significantly across the training conditions, with a trend to match these conditions. An analysis of variance (ANOVA) was carried out with age (4: years $6,8,10$, and adults) and training (2: 20\% SRP, 80\% SRP) as the between-subjects factors and starting location (2: top or bottom) as the withinsubjects factor. Figure 1 presents the mean percentages of respect for the principle as a function of age, training, and starting location.

As is shown in Figure 1, the rate at which participants had seen figures drawn in conformity with the principle had a significant impact on their further drawing behavior $[F(1,186)=18.21, p<.01]$. The participants who received $20 \%$ SRP training applied the principle in $37.03 \%$ of the test trials, whereas the performance value attained in the other training group was $59.07 \%$. The impact of observational training was the same irrespective of the starting location $[F(1,186)<1]$. More interesting, the age $\times$ training interaction did not reach significance $[F(3,186)=$ $1.34, p>.25$ ]. A planned comparison between children and adults for this interaction revealed that the children did not significantly differ from the adults $[F(1,186)=$ $1.17, p>.25]$.

\section{Explicit Knowledge}

The questionnaire phase showed that the adults did not differ as a function of their training condition when they were asked about what they might have perceived as regular in the training phase. Most of the participants reported that they saw four types of figures being drawn with a variable velocity and sometimes from the top, sometimes from the bottom. Table 1 displays the number of participants by group who reported the different judgments concerning how starting points and movement rotations were combined in the execution of the figures during the training phase.

When required to remember in which direction the figure was drawn when it started at the top or at the bottom (column "awareness about the manipulated covariation"
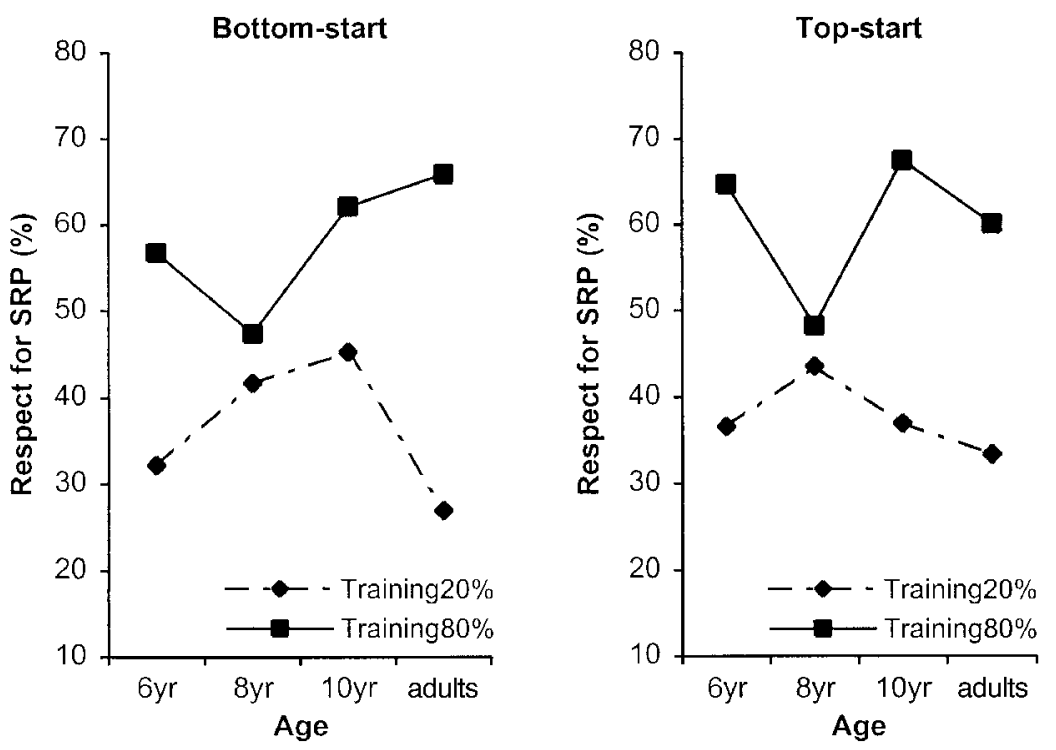

Figure 1. Mean percentage of respect for the SRP as a function of age, training, and starting location (top start and bottom start). 
Table 1

Number of Participants (Percentages in Parentheses) as a Function of Training and Age for the Different Explicit Responses Expressed in the Questionnaire

\begin{tabular}{|c|c|c|c|c|c|c|c|c|c|}
\hline \multirow[b]{2}{*}{ Training } & \multirow[b]{2}{*}{ Age } & \multirow[b]{2}{*}{$N$} & \multicolumn{4}{|c|}{$\begin{array}{l}\text { Dominant Combinations of } \\
\text { Starting Point/Rotation }\end{array}$} & \multicolumn{3}{|c|}{$\begin{array}{c}\text { Awareness About } \\
\text { the Manipulated Covariation }\end{array}$} \\
\hline & & & Top and Clock & Top and Cclock & Bottom and Clock & Bottom and Cclock & Correct & Mixed & Inverted \\
\hline \multirow[t]{4}{*}{$80 \%$ SRP } & 6 year & 21 & $8(38.1)$ & $13(61.9)$ & $13(61.9)$ & $8(38.1)$ & $7(33.3)$ & $12(57.1)$ & $2(9.5)$ \\
\hline & 8 year & 19 & $11(57.9)$ & $8(42.1)$ & $9(47.4)$ & $10(52.6)$ & $4(21.1)$ & $9(47.4)$ & $6(31.6)$ \\
\hline & 10 year & 22 & $10(45.4)$ & $12(54.5)$ & $12(54.5)$ & $10(45.4)$ & $5(22.7)$ & $14(63.6)$ & $3(13.6)$ \\
\hline & adults & 33 & $16(48.5)$ & $17(51.5)$ & $18(54.5)$ & $15(45.4)$ & $10(30.3)$ & $15(45.4)$ & $8(24.2)$ \\
\hline \multirow[t]{4}{*}{$20 \%$ SRP } & 6 year & 21 & $13(61.9)$ & $8(38.1)$ & $7(33.3)$ & $14(66.6)$ & $8(38.1)$ & $11(52.4)$ & $2(9.5)$ \\
\hline & 8 year & 22 & $12(54.5)$ & $10(45.4)$ & $6(27.3)$ & $16(76.2)$ & $8(36.4)$ & $12(54.5)$ & $2(9.1)$ \\
\hline & 10 year & 21 & $14(66.6)$ & $7(33.3)$ & $13(61.9)$ & $8(38.1)$ & $4(19.1)$ & $12(57.1)$ & $5(23.8)$ \\
\hline & adults & 35 & $19(54.3)$ & $16(45.7)$ & $17(48.6)$ & $18(51.4)$ & $11(31.4)$ & $15(42.8)$ & $9(25.7)$ \\
\hline
\end{tabular}

Note-Clock, clockwise rotation; Cclock, counterclockwise rotation. It is important to keep in mind that the judgment of correctness regarding the manipulated covariation differs according to the training condition. For the $80 \%$ group, it is correct if the participants expressed a judgment corresponding to the SRP, whereas for the $20 \%$ group, it is correct if the participants expressed a judgment corresponding to the inverse of the SRP, in conformity with the covariation they were exposed to.

in Table 1), 21 out of 68 adults reported correct covariation judgments (i.e., judgments conforming to the covariation they were exposed to), although slightly fewer (17 out of 68 ) expressed inverted judgments. No significant differences appeared between these two judgments, irrespective of whether the training condition was considered or not (Chi tests, $p \mathrm{~s}>.40$ ). Just as those obtained from adults, the free reports from the children did not reveal much about their awareness of the different regularities introduced in the training phase. However, in response to the question that directly tapped their explicit knowledge about whether or not the figures drawn on the screen conformed to the principle, the children demonstrated globally a greater degree of awareness than that shown by the adults. If we pool the children across age and training, 36 out of 126 reported correct judgments, and 20 out of 126 formulated incorrect judgments $\left[\chi^{2}(1)=5.88, p<.05\right]$. This tendency to report correct covariation judgments was significant at 6 years $\left[\chi^{2}(1)=8.23, p<.01\right]$, but not at the older ages $(p \mathrm{~s}>.30)$. A certain degree of awareness was still observed at 8 years; the children trained in the $20 \%$ SRP group also more frequently reported correct judgments (8 out of 22) than incorrect ones [2 out of 22; $\left.\chi^{2}(1)=4.66, p<.05\right]$. By contrast, the 10 -year-old children did not show any significant awareness of the relevant aspects of the training situation.

\section{Relationship Between Implicit Performance and Explicit Knowledge}

Although no clear-cut relationship emerged between explicit knowledge and the type of observational training received by the participants, at least in the adults, more subtle relationships may exist between their own performance in the test and the explicit knowledge they expressed in the questionnaire. In the Willingham et al. (1989) study for instance, important between-subjects differences were reported in the type of association between implicit and explicit knowledge. In our study, the participants from each training group were divided into three groups as a function of their explicit judgments about the manipulated covariation (1, judgment correct; 2 , mixed judgment; 3 , judgment incorrect), and their mean degree of respect for the principle during the implicit test was computed. Table 2 presents the results as a function of training and age. A positive relationship between performance and explicit judgment should be evidenced by a high degree of respect for the SRP in the implicit test in the $80 \%$ SRP group when correct judgments are reported and by a low degree of respect for the principle in the $20 \%$ SRP group when correct judgments are reported.

Such a positive relationship does, in fact, tend to emerge in the $80 \%$ SRP group. The adults who reported correct explicit judgments were also those who demonstrated the highest degree of respect for the principle in the test (79.6\%). This percent of respect fell to $57.5 \%$ and $52.6 \%$, respectively, for participants expressing either a mixed awareness or an incorrect judgment. A $t$ test showed that the difference was nearly significant $[t(31)=1.92, p=$ .06]. The same results emerged in children (Table 2A): Those who expressed correct covariation judgments achieved a higher implicit performance (respect for the principle in $78.6 \%$ of the cases) than those who had a mixed or incorrect judgment $(51.1 \%$ of respect) $[t(60)=$ $2.72, p<.01]$. The results were much less clear in the $20 \%$ SRP groups (Table 2B). In the adults, as in the children, there were no significant relationships between achieved implicit performance and expressed explicit judgments $(t$ tests, $p s>.14)$.

Finally, the participants who gained a correct level of explicit knowledge were omitted from the analysis. An ANOVA was run on the remaining participants, respectively, 14 and 13 (80\% SRP group and 20\% SRP group) 6-year-old children, 15 and 14, 8-year-old children, 17 and 17, 10-year-old children, and 23 and 24 adults. The effect of training was still significant $[F(1,129)=8.23, p<$ $.01]$, with the participants trained at $20 \%$ respecting the 
principle in $34.1 \%$ of the cases, whereas those trained at $80 \%$ showed a mean percent of respect of $52 \%$. These percentages are very close to those obtained when the whole set of data is analyzed. No other factor or interaction reached significance. In particular, the age $\times$ training interaction was still not significant $[F(3,129)<1]$. These results are in line with those reported by Burke and Roodenrys (2000), who recently showed that participants modified their behavior in an implicit learning task whether they developed relevant explicit knowledge or not.

\section{DISCUSSION}

The results reveal that learning a new motor behaviorimplicitly through observational training was efficient in adults and in children, when a procedure devised to avoid explicit influences on behavior was used. The impact of observational learning was not due to the fact that some participants gained an explicit knowledge of the relevant learning features. Even when these participants were withdrawn, the impact of learning was still significant. There was thus no necessary relationship between implicit performance and explicit knowledge as it is usually found in implicit learning tasks (i.e., by asking participants to remember certain relevant characteristics of the training situation). This explicit memory of the previous situation might be partial, or might be affected by certain strategic activities. In any case, it is not a necessary concomittant of implicit behavioral changes. The 6-year-olds reported the greatest number of correct explicit judgments. This might have been due to the fact that these young children naturally paid attention to such parameters as starting location and movement rotation in drawing because these parameters are constantly focused on during handwriting exercises. This enhanced attention would be specific to this age period.

Because the present results parallel those obtained when motor training was involved (Vinter \& Perruchet,
1999, 2000), it could be suggested that the motor component is not strictly necessary for implicit motor learning. However, it could be objected that the motor component was not actually eliminated in our task because the participants were asked to keep track of the moving point tracing the figure on the screen. Pursuit eye movements were therefore elicited. We admit that this point should urge us to make a cautious conclusion as far as the role of the motor component in observational implicit learning is concerned, though Vogt (1995) has demonstrated that learning by observation or by motor practice remains similar when eye movements are not required. However, Vogt's study concerned explicit learning, and the role of eye movements could be different in explicit and implicit observational learning. If we cannot ascertain that pure observational learning operated in our experiment, we can suggest that implicit motor learning does not require an overt motor practice. This conclusion is much in line with a large body of research that has established that strong relationships exist between perception, action, and mental imagery (e.g., Goodale \& Milner, 1992; Jeannerod, 1997; Prinz, 1997). However, we are not claiming that motor practice is useless in implicit motor learning. We suspect that it depends on the level at which the motor parameter used in the implicit motor task is encoded. Movement direction was the crucial parameter in our task, and this parameter seems to be encoded during motor preparation - that is, at a hierarchically higher level than other parameters like amplitude for instance, which also involves the motor execution phase (e.g., Georgopoulos, Lurito, Petrides, Schwartz, \& Massey, 1989). If amplitude is the crucial parameter for assessing implicit learning, a greater advantage might be obtained for motor than for observational training. Thus, in an SRT task in which participants have to produce movements adjusted both in direction and in amplitude with regard to target displacements, the role of the motor component might prove to be much greater than that observed in our drawing task.

Table 2

Mean Percentage and Standard Deviations of Respect for the Principle in the Implicit Test as a Function of Training, Age, and Explicit Knowledge

\begin{tabular}{|c|c|c|c|c|c|c|}
\hline \multirow[b]{3}{*}{ Age } & \multicolumn{6}{|c|}{ Training Set at $80 \%$ SRP } \\
\hline & \multicolumn{2}{|c|}{$\begin{array}{l}\text { Correct Judgment: } \\
\text { Conform to the SRP }\end{array}$} & \multicolumn{2}{|c|}{ Mixed Judgment } & \multicolumn{2}{|c|}{$\begin{array}{l}\text { Incorrect Judgment: } \\
\text { Inversion of the SRP }\end{array}$} \\
\hline & $M$ & $S D$ & $M$ & $S D$ & $M$ & $S D$ \\
\hline 6 years & 72.6 & 24.7 & 54.2 & 38 & 58.3 & 17.7 \\
\hline 8 years & 88.5 & 17.8 & 39.3 & 39.2 & 33.3 & 35 \\
\hline 10 years & 79.2 & 29.2 & 66.1 & 40.8 & 34.7 & 17.3 \\
\hline \multirow[t]{4}{*}{ Adults } & 79.6 & 23.6 & 57.5 & 33.1 & 52.6 & 42.2 \\
\hline & \multicolumn{6}{|c|}{ Training Set at $20 \%$ SRP } \\
\hline & \multicolumn{2}{|c|}{$\begin{array}{l}\text { Correct Judgment: } \\
\text { Inversion of the SRP }\end{array}$} & \multicolumn{2}{|c|}{ Mixed Judgment } & \multicolumn{2}{|c|}{$\begin{array}{l}\text { Incorrect Judgment: } \\
\text { Conform to the SRP }\end{array}$} \\
\hline & $M$ & $S D$ & $M$ & $S D$ & $M$ & $S D$ \\
\hline 6 years & 38.5 & 38.4 & 35.6 & 40 & 10.4 & 14.7 \\
\hline 8 years & 56.8 & 38.9 & 32.3 & 35.4 & 47.9 & 8.8 \\
\hline 10 years & 57.3 & 47.2 & 34.7 & 37 & 43.3 & 49 \\
\hline Adults & 24.2 & 21.1 & 33.6 & 35.2 & 31.5 & 20.6 \\
\hline
\end{tabular}


The impact of observational motor implicit learning did not differ between the children and the adults. However, if observational learning tended to elicit the intervention of an explicit system of knowledge (e.g., Rüsseler \& Rösler, 2000), the young children should have been disadvantaged compared with the adults, because of their better ability to rely on procedural rather than on declarative knowledge (Karmiloff-Smith, 1992). We suggest that a more basic form of perceptual learning than that elicited in earlier studies could be implemented in our task. In earlier studies, an explicit search for rules or regularities in the training situation could have been elicited simply because the participants were free to engage in such a process during observation (they were not required to do anything else than to observe). The type of learning process activated in our task is probably similar to the associative form of perceptual learning that Mandler $(1988,1992)$ distinguished from another type that she termed perceptual analysis mechanism. Associative perceptual learning is automatic, accomplished through repetitions, sensitive to frequency and surface features, and sustains abilities like motor skill learning or perceptual categorization. By contrast, the perceptual analysis mechanism operates on the products of attentive processing, selects relevant structural features and redescribes what is perceived into more abstract representations that form the basic units for explicit knowledge. We suggest that it is this perceptual analysis mechanism that is predominantly activated when participants are simply required to observe in the absence of any other instructions. This mechanism is much more prone to failures in learning than is the automatic associative form because it possesses a highly strategic component.

In conclusion, the present study provides evidence for the efficiency of implicit motor learning by observation. However, as suggested above, the generalization of this result could be limited, because the necessity of the motor component could depend on the crucial movement parameter selected in the study and because different types of observation could produce different results, depending on whether they are prone to elicit an explicit perceptual analysis mechanism.

\section{REFERENCES}

Burke, D., \& Roodenrys, S. (2000). Implicit learning in a simple cued reaction time task. Learning \& Motivation, 31, 364-380.

Cleeremans, A. (1993). Mechanims of implicit learning: A connectionist model of sequence processing. Cambridge, MA: MIT Press, Bradford Books.

Cohen, A., Ivry, R., \& Keele, S.W. (1990). Attention and structure in sequence learning. Journal of Experimental Psychology: Learning, Memory, \& Cognition, 16, 17-30.

Georgopoulos, A. P., Lurito, J. T., Petrides, M., Schwartz, A. B., \& MASSEY, J. T. (1989). Mental rotation of neuronal population vector. Science, 243, 234-236.
Goodale, M. A. \& Milner, A. D. (1992). Separate visual pathways for perception and action. Trends in Neurosciences, 15, 20-25.

Howard, J. R., Mutter, S. A. \& Howard, D. V. (1992). Serial pattern learning by event observation. Journal of Experimental Psychology: Learning, Memory, \& Cognition, 18, 1029-1039.

JEANNEROD, M. (1997). The cognitive neuroscience of action. Oxford: Blackwell.

Karmiloff-Smith, A., (1992). Beyond modularity: A developmental perspective on cognitive science. Cambridge, MA: MIT Press.

MANDLER, J. M. (1988). How to build a baby: On the development of a representational system. Cognitive Development, 3, 113-136.

MANDLER, J. M. (1992). How to build a baby: II. Conceptual primitives. Psychological Review, 99, 587-604.

Maybery, M., Taylor, M., \& O'Brien-Malone, A. (1995). Implicit learning: Sensitive to age but not IQ. Australian Journal of Psychology, 47, 8-17.

Natt Kemper, D., \& Prinz, W. (1997). Stimulus and response anticipation in a serial reaction task. Psychological Research, 48, 231-238.

NisSEN, M. J., \& BULLEMER,P. (1987). Attentional requirements of learning: Evidence from performance measures. Cognitive Psychology, 19, 1-32.

Perruchet, P., \& Vinter, A. (1998). Learning and development. The implicit knowledge assumption reconsidered. In M. Stadler \& P. Frensch (Eds.), Handbook of implicit learning (pp.495-531). Thousand Oaks, CA: Sage.

PEw, R.W. (1974). Levels of analysis in motor control. Brain Research, 71, 393-400.

Prinz, W. (1997). Perception and action planning. European Journal of Cognitive Psychology, 9, 129-154.

REBER, A.S. (1993). Implicit learning and tacit knowledge: An essay on the cognitive unconscious. New York: Oxford University Press.

RüSSELER, J., \& RöSlER, F. (2000). Implicit and explicit learning of event sequences: Evidence for distinct coding of perceptual and motor representations. Acta Psychologica, 104, 45-67.

STADLER, M. A. (1989). On learning complex procedural knowledge. Journal of Experimental Psychology: Learning, Memory, \& Cognition, 15, 1061-1069.

Van Sommers, P. (1984). Drawing and cognition. Cambridge: Cambridge University Press.

Vinter, A, \& Perruchet,P. (1999). Isolating unconsciousinfluences: The neutral parameter procedure. Quarterly Journal of Experimental Psychology, 52A, 857-875.

Vinter, A., \& Perruchet, P. (2000). Unconscious learning in children is not related to age: Evidence from drawing behavior. Child Development, 71, 1223-1240.

ViViani, P., \& STUCCHI, N. (1992). Motor-perceptual interactions. In G. E. Stelmach \& J. Requin (Eds.), Tutorials in motor behavior II (pp. 229-248). Amsterdam: North-Holland.

VoGT, S. (1995). On relations between perceiving, imagining and performing in the learning of cyclical movement sequences. British Journal of Psychology, 86, 191-216.

WillinghaM, D. B. (1999). Implicit motor sequence learning is not purely perceptual. Memory \& Cognition, 27, 561-572.

Willingham, D. B., Nissen, M. J., \& Bullemer, P. (1989). On the development of procedural knowledge. Journal of Experimental Psychology: Learning, Memory, \& Cognition, 15, 1047-1060.

WULF, G., \& SCHMIDT, R. A. (1997). Variability of practice and implicit motor learning. Journal of Experimental Psychology: Learning, Memory, \& Cognition, 23, 987-1006.

ZiESSLER, M. (1994). The impact of motor responses on serial-pattern learning. Psychological Research, 57, 30-41.

(Manuscript received April 20, 2001;

revision accepted for publication November 14, 2001.) 